\title{
Content-aware Network Coding for Layered Video over Wireless Relay Networks
}

\author{
Yong-woo Lee, Tae-min Cho, and Jitae Shin \\ School of Electronic and Electrical Eng., Sungkyunkwan University, Suwon, Rep. of Korea \\ \{tencio2001, discross, jtshin\}@skku.edu
}

\begin{abstract}
Transmission issue of wireless video, as the hugest percentage among mobile traffics, is a challenge problem in wireless/mobile networks. To tackle video transmission problem occurred in heterogeneous networks as well as different devices, scalable video coding (SVC) has been developed. Also, network coding (NC) enables to transmit efficiently for same given bandwidth. In this paper, we propose a simple random linear network coding (RLNC) for SVC transmission with decode and forward (DF) system over wireless relay network. In order to transmit base layer of SVC with high reliability using characteristics of RLNC in DF relay system, we propose a method which re-groups differently basic coding units of base layer and enhancement layers in SVC. The simulation shows our proposed method gains better performance compared to previous methods.
\end{abstract}

Keywords - Network coding (NC), Scalable Video Coding (SVC), Relay network, Decode and forward, Amplify and forward

\section{INTRODUCTION}

Today, with popularization of smart devices, data traffic has proliferated in wireless network. With this trend, voice traffics that took a large portion in wireless network have become minor issue; on the other hand, the demand and supply for video traffics have become tremendous issue and taken over the place. Therefore, in order to reduce the traffic load, one should deal with video transmission methods that can provide good quality and reliability.

Network coding (NC) has been designed for wired networks that use multicast protocol to have maximum capacity [1]. In previous data transmission scheme, relay only serves as a routing role. Instead, NC allows the relay to code the data so that more data can be transmitted to next nodes. Later on, there are many attempts to use NC not only in wired networks, but also wireless networks. In addition to these attempts, with the development of random linear network coding (RLNC) [2], one can apply NC to dynamic topologies.

H.264/AVC and HEVC use fixed bitrate to transmit video data which is not efficient when it comes to heterogeneous network where bandwidth or capacity is different. To tackle this problem, the scalable extension of H.264/AVC (SVC) has been designed. With this codec, one can divide a video bit stream into a base layer and enhancement layers based on temporal, spatial, and quality criteria. The SVC [3] consists of base layer (BL) and enhancement layers (ELs). The BL contains the basic information of data that guarantees minimum quality of video and the ELs contain additional information. The more ELs are obtained, the better quality can be derived on the basis of BL. User equipment receives a BL and several ELs according to the network condition, thus one can receive the best video data in the user environment. However, since the ELs have dependency upon the BL, it is no use when BL information is lost. Therefore, it should be addressed that BL should be regarded as first priority.

The rest of this paper is organized as follows. Previous works regarding $\mathrm{NC}$ are treated in Section 2. Our proposed algorithm is introduced in Section 3. The performance of simulation results is evaluated in Section 4. Finally, conclusion of this paper is given in Section 5.

\section{Previous Works}

Issues about transmitting SVC video using contemporary $\mathrm{NC}$ are listed and pros and cons of them are analyzed.

\section{A. Hierarchical Network Coding}

Hierarchical network coding (HNC) is a method to protect more important data in SVC video scheme. Nguyen et al. [4] have proposed a method to have protection for base layer by RLNC. In this method, the dependent layer is included when it comes to encode one layer, thus, the dependent layer is strongly protected. Limmanee et al. [5] also proposed a method to protect dependent layer using RLNC that is similar to Nguyen et al. Vukobratovic et al. [6] proposed a method that has stronger protection upon dependent layer by using expanding window. These methods may have gains over rateless coding streaming environments.

However, since they send additional dependent layers, they may consume unnecessary additional capacities when it comes to transmitting video data in a general way.

\section{B. Layer Based Network Coding}

Layer based network coding is a method that uses NC to transmit video data in the same layer. Mirshokraie et al. [7] proposed a method to transmit SVC video data over P2P streaming environment. Based on RLNC, it decides specific layers to transmit adaptively according to peer's network condition. In comparison with $\mathrm{HNC}$ mentioned before, it is advantageous to use less capacity as well as to transmit SVC video data toward heterogeneous devices in terms of video transmission. 
Nyugen et al. [8] designed a streaming system based on RLNC considering SVC and network condition named Chameleon. It makes one packet out of several segments in the same layer. Then it is transmitted to destination node by RLNC. In case of the condition of destination is worse, i.e. the bandwidth is deficient, it reduces one layer being transmitted to make sure a certain quality of video data received in the destination node. Layer based NC shows better performance with respect to network quality index such as buffer time and delay compared to contemporary video streaming algorithms.

However, both methods do not consider the priority of BL over EL so that the protection is always same upon both layers.

\section{III.PRoposed Algorithms}

In this section, we introduce the reason why DF network system is reasonable to RLNC in relay network and analyze the performance of decode-and-forward (DF) and amplifyand-forward (AF). Using the results, we propose an algorithm to transmit base layer with more reliability.

\section{A. DF Relay Network System}

If there are data that can be decoded in relay node, DF relay network system decodes the data and forwards again. It is very similar to AF relay network system as it stands for literally, but the performance role in relay node is completely different. After the decoding, it restores original packets and encodes using RLNC by picking coefficients out of a finite field. Segments that cannot be decoded in relay node are transmitted to the destination node as it is. The destination node decodes the data to restore original packets like any other AF relay network system does as long as it gets a certain amount of segments. Otherwise, the destination discards the segments or sends re-transmission request since it cannot decode the data.

In relay network, DF relay system is the way to reinforce RLNC. In this method, the key point is that it applies RLNC one more time in relay node to make more segment candidates that can be decoded. Suppose one packet consists of $n$ segments, receiver does not need to consider which segment it gets, but different kinds. In other words, it just needs to have $n$ segments that are linearly independent. Therefore, with DF, the probability to restore original packet gets higher.

\section{B. Re-grouping Generations within a Packet}

With only DF, it is very similar to layer based network coding such as Chameleon [8], which does RLNC to SVC video data in all nodes except it does RLNC only in relay node. Therefore, to enhance reliability of the most important information, i.e. BL, we propose an algorithm that re-groups generations in a packet unit.

Previous methods divide a packet into $n$ generations, and then it converts them to one encoded data with RLNC before transmission. Proposed algorithm has a thread of connection that it divides a packet into $n$ generations. However it does not convert them into one encoded data with RLNC, it converts them into two encoded data with RLNC, while the ELs maintain one encoded data unit.

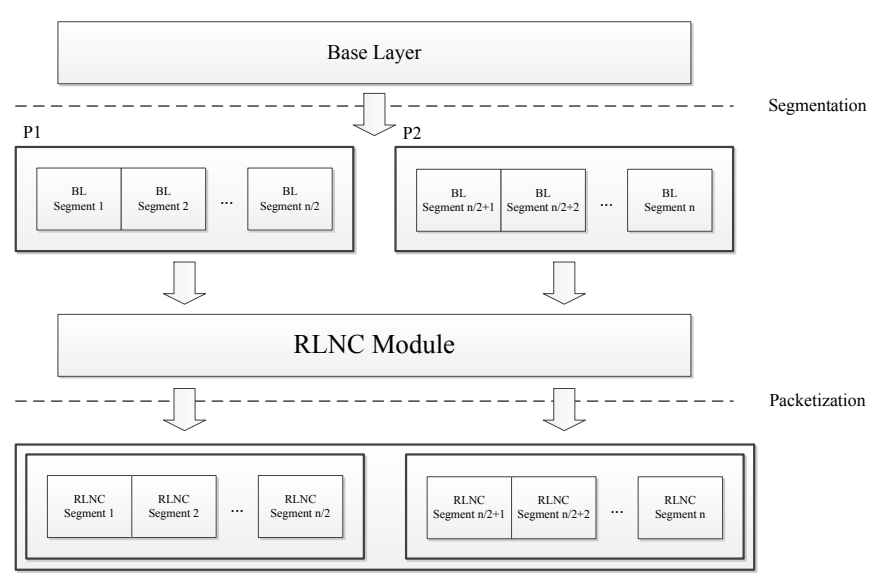

(a) RLNC of $\mathrm{BL}$

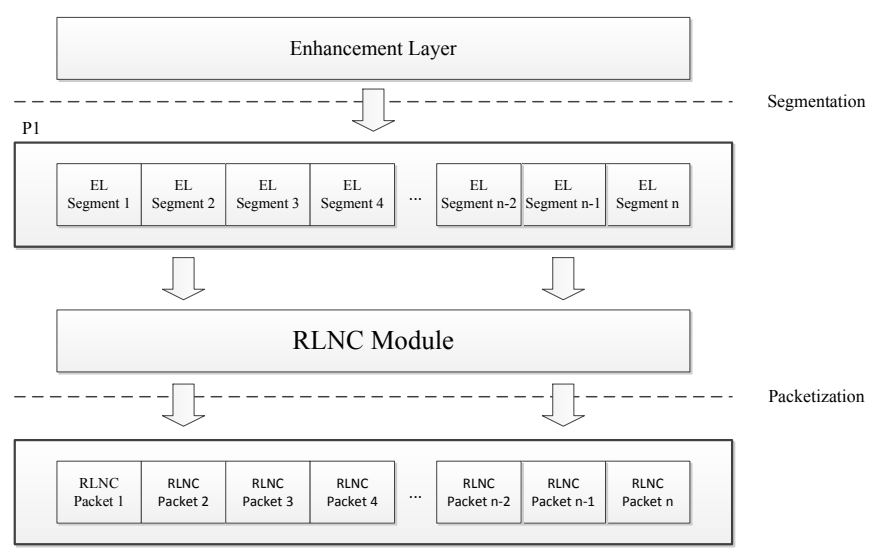

(b) RLNC for ELs

Figure 1. Proposed re-grouping algorithm of RLNC for SVC.

Fig. 1 shows the proposed application usage of RLNC. In previous algorithm if one segment in the packet is lost, the whole packet shall be discarded. In the proposed scheme, however, it is possible that one can restore the other group of segments among two groups shown in Fig. 1 (a), thus the probability to restore original packet gets even much higher. It seems that it is not different from just reducing the packet size. If one just reduces the packet size, it increases a great deal of the whole overhead size since the number of headers increases with the increase of the number of packets. On the other hand, the proposed algorithm divides a packet only logically with RLNC characteristic, the physical number of packet does not increase.

There is no help for adding slight information about regrouping into header. Thus, we apply the algorithm only to $\mathrm{BL}$, which has the most important information, to prevent the whole overhead size from increasing as in Fig. 1.

\section{IV.Simulation RESULTS}

We evaluate the proposed algorithm from two points of view. First, we make sure that DF relay system shows better performance compared to AF relay system which is currently 
used popularly. Next, we compare our proposed algorithm to contemporary RLNC method based on SVC.

Table 1 shows network environment used in this simulation and topology is presented in Fig. 2. Used video bitstream for transmission is encoded to have one base layer and two enhancement layers by Joint Scalable Video Model (JSVM) 9.19 reference software and open SVC decoder is used to restore the video bitstream that have lost some packets during transmission.

TABLE 1. Simulation Settings

\begin{tabular}{|c|c|}
\hline \multicolumn{2}{|c|}{ Video Encoder Settings } \\
\hline SVC Encoder & H.264/SVC JSVM 9.18 \\
\hline SVC Decoder & Open SVC \\
\hline Sequence Name & FOREMAN \\
\hline Image Format & CIF $(352 \times 288)$ \\
\hline Frame Rate & 30 [frames per second] \\
\hline Number of Encoded Frames & 150 \\
\hline MATLAB Version & $2013 a$ \\
\hline Encoding Method & $1 \mathrm{BL}, 2$ SNR ELs \\
\hline \multicolumn{2}{|c|}{ Channel Settings } \\
\hline S-D:S-R:D-R Link Distance & $\sqrt{2}: 1: 1$ \\
\hline Channel Error & Random Error \\
\hline
\end{tabular}

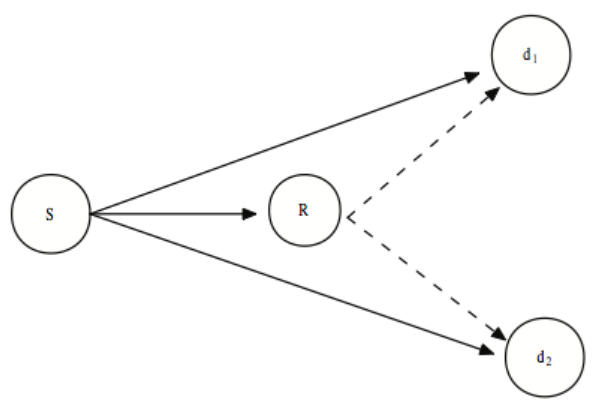

Figure 2. Wireless relay network topology

\section{A. DF Relay System Evaluation}

The simulation evaluates three cases, without using network coding, AF relay system with RLNC, and DF relay system with RLNC.

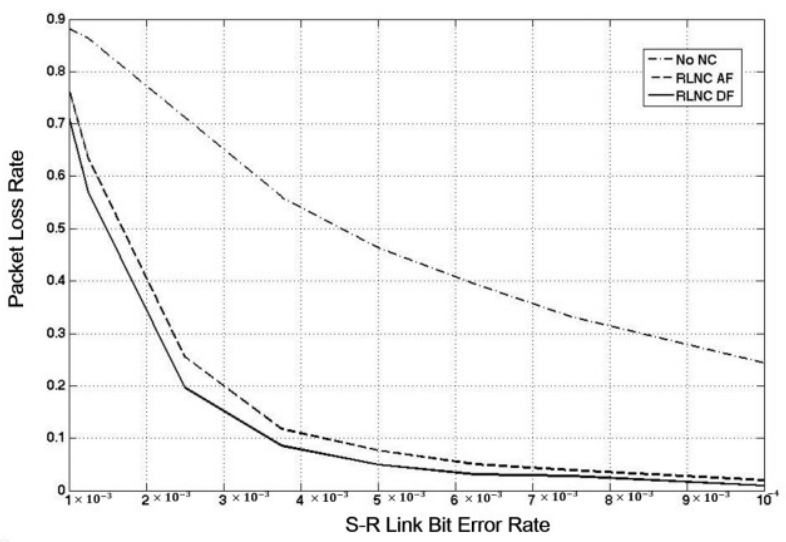

Figure 3. Performance comparison of DF and AF relay system
Fig. 3 shows the cases with RLNC have tremendously better performance than methods without using network coding. Also, in comparison of AF with RLNC and DF with RLNC, DF with RLNC gains better performance maximum about $7 \%$. As a result, as expected, DF with RLNC is more suitable for relay network system.

\section{B. Video Quality Evaluation}

In addition to the simulation, we compare our proposed method to previous layered network coding, Chameleon [8]. Fig. 5 shows our proposed method. As expected, with RLNC characteristics, we can enjoy effectiveness of re-grouping generations in a packet with a little additional overhead. Especially, under worse channel condition, the difference gets bigger which is presented in Fig. 4. Lastly, we show the YPSNR comparison in Fig. 5. The result shows the restored data have better PSNR performance compared to previous method, i.e. Chameleon.

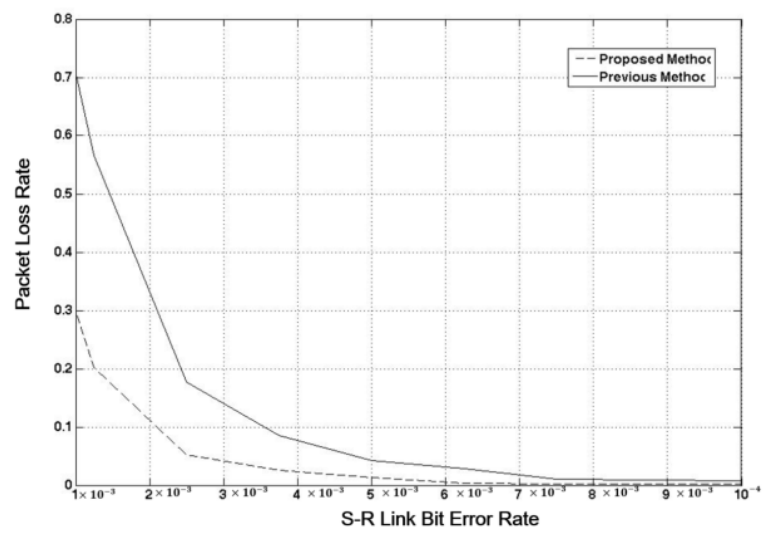

Figure 4. Performance comparision of proposed and previous methods

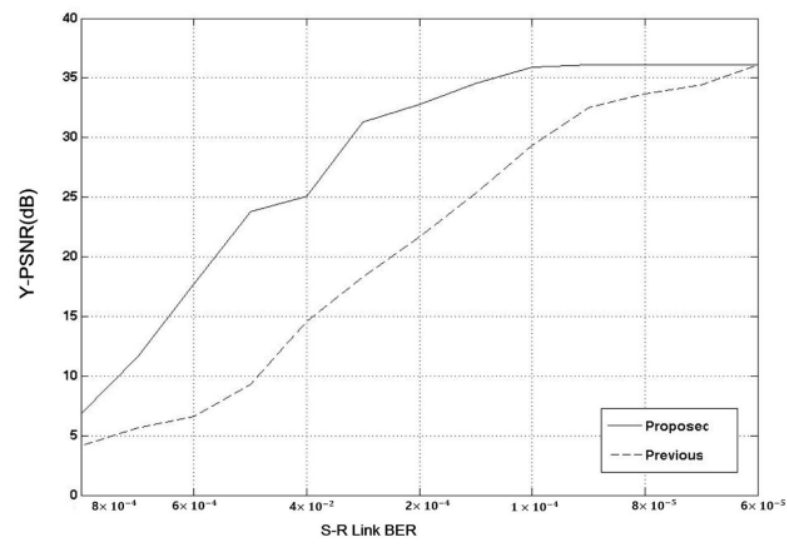

Figure 5. Y-PSNR comparision of proposed and previous methods

\section{Conclusions}

In this paper, we propose an efficient network coding algorithm for SVC video transmission in wireless DF relay system. First of all, we compare DF relay system with RLNC to AF relay system with RLNC and showed the reason why DF is suitable for relay network. DF had maximum about $7 \%$ gains over AF. Secondly, we re-group generation within a 
packet into fragmented sub-groups and show the performance with previous method, Chameleon. Although, the proposed algorithm cannot help increasing slight overhead, we minimize the increasing size of overhead by only applying upon BL. The performance comparison show the proposed algorithm has better performance over previous layer-based network coding.

\section{ACKNOWLEDGMENT}

This research was funded by the MSIP (Ministry of Science, ICT \& Future Planning), Korea in the ICT R\&D Program 2013.

\section{REFERENCES}

[1] R. Ahlswede, N. Cai, S. Y. R. Li, and R. W. Yeung, "Network information flow," IEEE Transaction on Information Theory, vol. 46, pp. 1204-1216, July 2000.

[2] T. Ho, M. Medard, R. Koetter, D. R. Karger, M. Effros, J. Shi, and B. Leong, "A random linear network coding approach to multicast," IEEE Transactions on Information Theory, vol. 52, pp. 4413-4430, Oct. 2006.

[3] H. Schwarz, D. Marpe, and T. Wiegand, "Overview of the scalable video coding extension of the H.264/AVC standard," IEEE Transaction of Circuits and Systems for Video Technology, vol. 17, pp. 1103-1120, Sept. 2007.

[4] K. Nguyen, T. Nguyen, and S. Cheung, "Peer-to-peer streaming with hierarchical network coding," in Proc. ICME, 2007, pp. 396-399.

[5] A. Limmanee and W. Henkel, "UEP network coding for scalable data", in Proc. Turbo Codes and Related Topics, 2008, pp. 333-337.

[6] D. Vukobratovic and V. Stankovic, "On unequal error protection random linear coding for scalable video broadcasting," in Proc. Packet Video Workshop (PV), 2010, pp. 48-55.

[7] S. Mirshokraie and M. Hefeeda, "Live peer-to-peer streaming with scalable video coding and network coding," in Proc. MMSys, 2010, pp. 123-132.
[8] A. T. Nguyen, B. Li, and F. Eliassen, "Chameleon: adaptive peer-topeer streaming with network coding," in Proc. INFOCOM, 2010, PP. $1-9$.

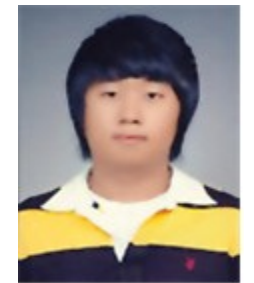

Yong-woo Lee received the B.S. degree from Sungkyunkwan University in 2013. He is currently a M.S. candidate in the Department of Electronic, Electrical and Computer Engineering, College of Information and Communication Engineering, Sungkyunkwan University, Rep. of Korea. His research interests include video signal processing and transmission over next generation Internet and wireless/mobile networks, $5 \mathrm{G}$ communication systems, and medical image processing.

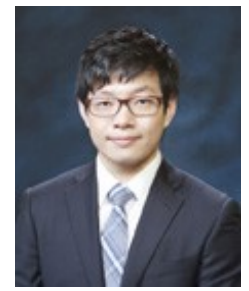

Tae-min Cho received the B.S. degree from Sungkyunkwan University in 2012. He is currently a M.S. candidate in the Department of Electronic, Electrical and Computer Engineering, College of Information and Communication Engineering, Sungkyunkwan University, Rep. of Korea. His research interests include video signal processing and transmission over next generation Internet and wireless/mobile networks, $5 \mathrm{G}$ communication systems, compressive sensing, and network coding.

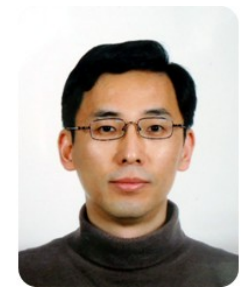

Jitae Shin received the B.S. degree from Seoul National University in 1986, the M.S in Korea Advanced Institute of Science and Technology (KAIST) in 1988. After 8 years working in Korea Electric Power Corp. (KOPEC) and Korea Atomic Energy Research Institute (KAERI), he returned to study and received the M.S. and Ph.D. degrees in Electrical Engineering from University of Southern California, Los Angeles, U.S.A. in 1998 and 2001, respectively. He is a Professor in College of Information and Communication Engineering of Sungkyunkwan University, Suwon, Korea. His research interest includes video signal processing and transmission over next generation Internet and wireless/mobile networks, 5G communication systems, and multimedia network control/protocol issues. 